\title{
Endoscopic retrieval of ingested magnets in children and role of magnetic retrievers
}

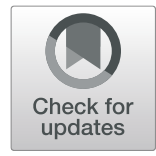

\author{
Wael M. Elzeneini ${ }^{*}$ (D) and Eleri Cusick
}

\begin{abstract}
Background: Various high-powered magnetic toy sets constitute a major hazard as the small magnets can be easily swallowed or aspirated by young children. Multiple ingested magnets or the ingestion of a magnet with another metallic object can pose a serious threat as this may lead to bowel perforation, fistulation, intestinal volvulus, bowel obstruction and death.

Main body: Retrieval of ingested magnets from the stomach is routinely performed endoscopically but can become a painstaking quest involving many tedious attempts to secure the magnet(s). Historically, pre-routine endoscopy, magnetic retrievers were used under fluoroscopic control for the removal of metallic objects in the stomach. With the advent of endoscopic retrieval instruments, they fell out of favour and are no longer readily available on the market. Despite this, conventional endoscopic instruments are likely to be less effective than endoscopic-assisted magnetic retrievers due to confounding weak bonds which form between the metal retrieval device and the magnet.

Preoperative testing, and selection of the retrieval instrument with the strongest coupling effect with a magnet, might be beneficial. A simple, endoscopic-assisted magnetic retriever created in theatre can allow for more effective, time-efficient and safe removal of ingested magnets under direct vision.

Conclusion: Urgent removal of multiple ingested magnets or a single ingested magnet coupled with another ingested metallic object should be highlighted. Stricter implementation of a widespread ban over loose magnetic toys is needed. Standardised protocols for the surgical management of ingested magnets should be available in every hospital.
\end{abstract}

Keywords: Magnetic retrievers, Ingested magnets, Foreign body ingestion, Endoscopy, Retrieval

\section{Background}

Foreign body ingestion is a very common presentation to any Accident \& Emergency Department dealing with children. The American Association of Poison Control Centres has found foreign body ingestion to be one of the five most common human exposures, accounting for almost 80,000 cases per year reported to poison control in children up to the age of 5 years [1]. Although magnet ingestion is still uncommon within this group, the Electronic Injury Surveillance System in USA revealed in

* Correspondence: wael.elzeneini@nhs.net

Department of Pediatric Surgery, Bristol Royal Children's Hospital, Upper Maudlin St, Bristol BS2 8BJ, UK

\section{Springer Open}

excess of 22,000 cases in children in the period of 2001 and 2011 [2].

Various high-powered magnetic toy sets such as Buckyballs and Buckycubes (Maxfield and Oberton Holdings, Brooklyn, NY), Magnetix (Mega Brands, Montreal, QC) together with tiny magnets within building sets, dolls and accessories as "Mattel's Polly Pocket Magnetic Play Sets" constitute a major hazard as they can be easily swallowed or aspirated by young children [3]. Recently, a new craze for fake tongue piercings with magnetic balls has prompted frequent incidents of them being swallowed with severe implications [4]. Multiple ingested magnets or the ingestion of a magnet with another metallic object can pose a serious threat as this

(c) The Author(s). 2021 Open Access This article is licensed under a Creative Commons Attribution 4.0 International License, which permits use, sharing, adaptation, distribution and reproduction in any medium or format, as long as you give appropriate credit to the original author(s) and the source, provide a link to the Creative Commons licence, and indicate if changes were made. The images or other third party material in this article are included in the article's Creative Commons licence, unless indicated otherwise in a credit line to the material. If material is not included in the article's Creative Commons licence and your intended use is not permitted by statutory regulation or exceeds the permitted use, you will need to obtain permission directly from the copyright holder. To view a copy of this licence, visit http://creativecommons.org/licenses/by/4.0/. 
may lead to bowel perforation, fistulation, intestinal volvulus, bowel obstruction and death [3, 5-8].

Efforts worldwide have recently been tried to limit this problem including various warning labels on magnetic toys, public warnings about the hazards of unintentional swallowing and inhalation of magnets in children and various awareness campaigns highlighting the potential dangers. Industrial standards have been changed to prevent detachment of loose magnets from magnetic toys aimed at children less than 14 years of age [9]. A further mass recall and ban from the market was held worldwide $[6,10]$. However, in spite of all these efforts, multiple studies have shown that magnet ingestion in children is still on the rise $[3,11,12]$. According to a survey carried out by the North American Society for Paediatric Gastroenterology, Hepatology, and Nutrition in 2012, warning labels and bans have failed to prevent magnet ingestion in children [13]. This may in part be explained by the increase in popularity of magnetic toys amongst children which still remain available online and many being sold as adults products such as Nanodots (Nano Magnetics, Ontario, Canada) [3].

\section{Main body}

Increased concerns regarding the morbidity and mortality associated with magnet ingestion has led to different algorithms and protocols set up to manage these cases. Ingestion of multiple magnets or a magnet and a second metallic object renders the patient at serious risk and mandates immediate removal either endoscopically if it is still in the stomach or through surgery whether laparoscopically or a laparotomy $[3,14,15]$.

Retrieval of ingested foreign bodies from the stomach has been routinely performed endoscopically utilising retrieval forceps, snare nets or stone retrieval baskets. With ingested magnets, however, endoscopic retrieval instruments may not work optimally as the ingested magnets may attach to the instrument's metallic rim immediately upon contact, preventing the retrieval instrument from closing securely upon the ingested magnets. It can become a painstaking quest involving many tedious attempts to secure the magnet(s). Moreover, the weak force with which the ingested magnet(s) attach to the metallic rim of the retrieving instrument renders them easily dislodged upon withdrawal of the endoscope. Contact with the upper and lower oesophageal sphincters is particularly troublesome. This leads to prolonged surgery time, increased surgical frustration and the possibility of the ingested magnets uncoupling during retrieval and obstructing the airway.

It is unclear from the literature how successful endoscopic removal of ingested magnets lying within the stomach is. While Richard et al. reports a success rate of $66 \%$ in general, it is unclear how much of that is due to technical difficulty in retrieving magnets from the stomach versus passage of some or more of the magnets further downstream [14]. The size and configuration of the ingested magnets has been described as one factor in unsuccessful endoscopic removal and need for laparotomy [16].

We found that by obtaining a sample of the ingested magnets from the parents and pre-operatively testing their coupling force with various endoscopic retrieval instruments, we could select the most effective retrieval instrument in advance. Different endoscopic retrieval instruments have different coupling force with magnets. In our experience with a string of ingested Buckyballs, we found that the biopsy forceps were more effective than the endoscopic grasper in keeping hold of the ingested magnets while retrieving them, despite the biopsy forceps being smaller in size. This may relate to the density of iron in the different instruments.

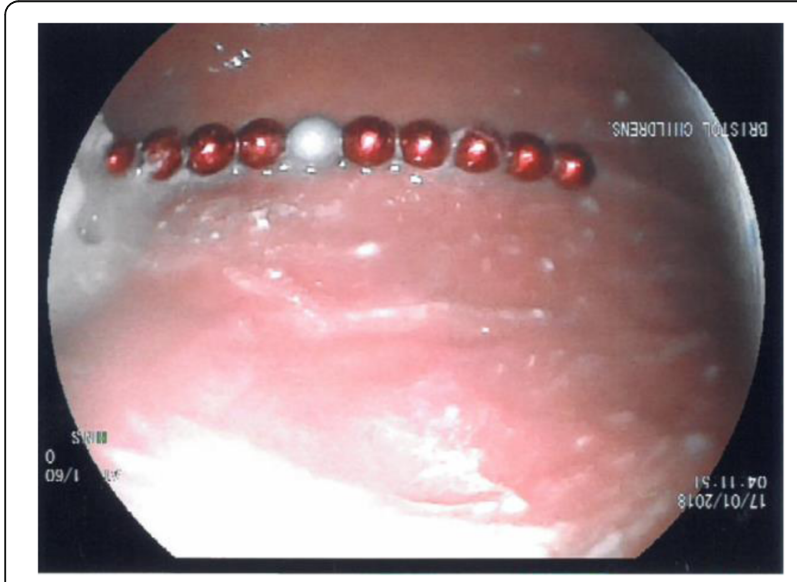

Fig. 1 Endoscopic image of a chain of 18 Buckyball magnets inside the stomach of a child

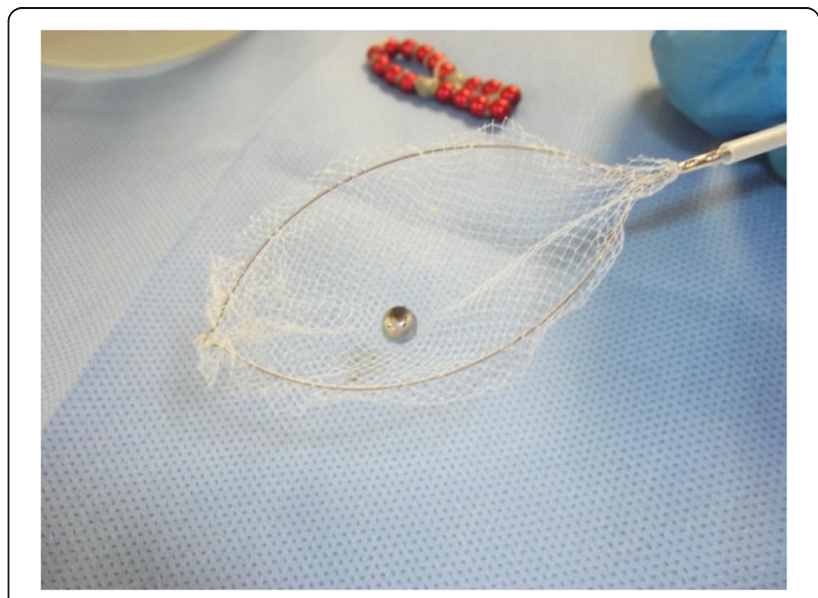

Fig. 2 One spare Buckyball magnet inserted into a retrieval net 

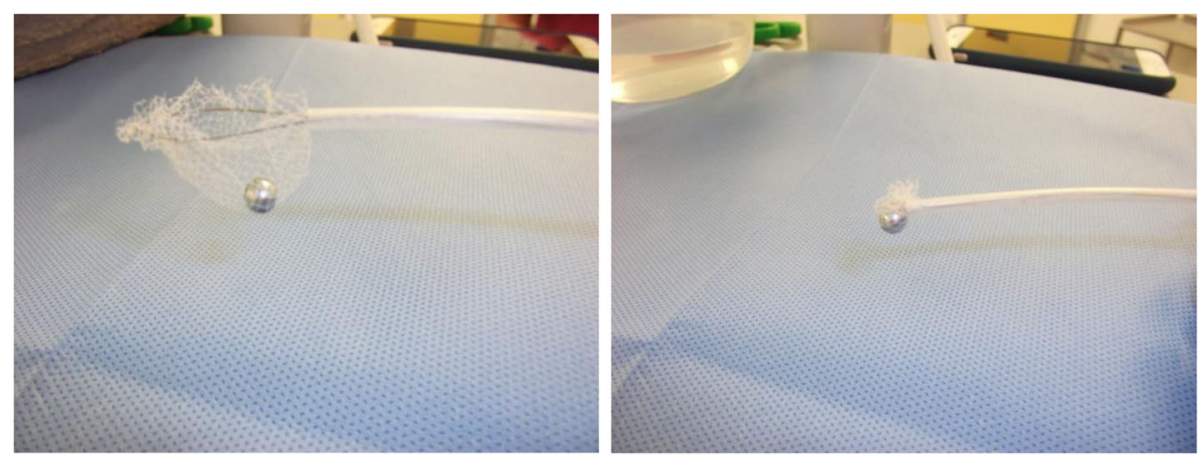

(A)

(B)

Fig. 3 A, B Retrieval net tightened to securely attach the Buckyball magnet to the retrieval net

Historically, magnetic retrievers were used for the removal of metallic objects in the stomach under fluoroscopy. They were formed of orogastric tubes that consisted of a long flexible tube with a strong magnet at its end. This was recognised to be a basic and effective form of retrieval for ingested metallic objects which was well tolerated awake and did not require any risks of surgery or general anaesthesia. However, it required the availability of fluoroscopy. Retrieval was carried out under fluoroscopic control rather than endoscopic vision and the potential risk of uncoupling of retrieved items and the inability to assess mucosal injury were seen as disadvantages [17-21]. With the recent advent of endoscopic retrieval instruments, magnetic retrieval devices fell out of favour and are no longer readily available on the market [22].

A few case reports previously described various ways of creating and utilising a primitive magnetic retriever under endoscopic guidance. They were used to retrieve either metallic or magnetic items from the stomach.

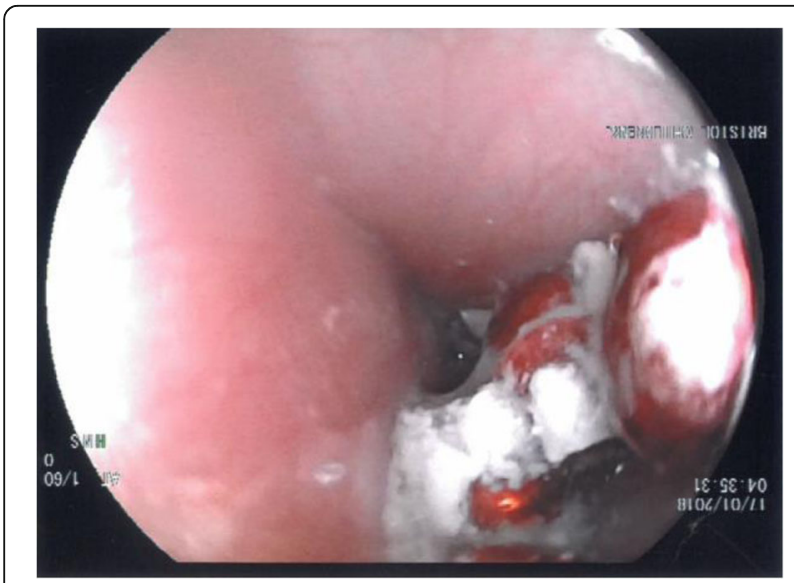

Fig. 4 Endoscopic extraction of the ingested Buckyball magnets using the basic magnetic retriever alongside endoscopic guidance
Two case reports were found in adults: one included a large refrigerator magnet placed into a Roth Net and tightened before insertion into the stomach [23], while the other involved a snared magnet that was filed previously to have 2 linear grooves for the snare to tighten upon [24]. Four other reports were found in children including a 12-Fr magnet head tube (Cook Co., Bloomfield, IL, USA) alongside a slim paediatric endoscope (OD $3.5 \mathrm{~mm}$ ) [25], a magnet stuck to the end of an endoscope [26], two magnetic disks connected by a steel spring and wire [27] and a magnetised loop basket [28].

We believe that magnetic retrievers have the immense advantage of having a strong magnetic force which couples with metallic objects or magnets, making it extremely hard for them to uncouple during their extraction. We have developed a simple way of creating a basic magnetic retrieval device that can be used alongside the paediatric endoscope or through the working channel to remove magnets located in the stomach in children. The retrieval process can be done safely and effectively under vision.

Our idea of an endoscopic-assisted magnetic retriever came during a failed endoscopic retrieval attempt of a long bead of 16 Buckyball magnets, measuring $9 \mathrm{~cm}$ in total length and lying within the stomach and duodenum (Fig. 1). Prior attempts with various conventional retrieval instruments including retrieval forceps, snare nets and retrieval nets failed as the ingested magnets repeatedly attached to the metallic rim of the instrument and which hence failed to close securely upon the ingested magnets. Moreover, it kept uncoupling while coming out with the endoscope as it was only attached to it with a weak magnetic force.

The basic magnetic retrieval device was created by placing a small magnet within an endoscopic retrieval net (Fig. 2). The retrieval net was then tightened onto the small magnet to secure it and make sure it stays enclosed within the retrieval net (Fig. 3). This was inserted under vision alongside (and not through) the 

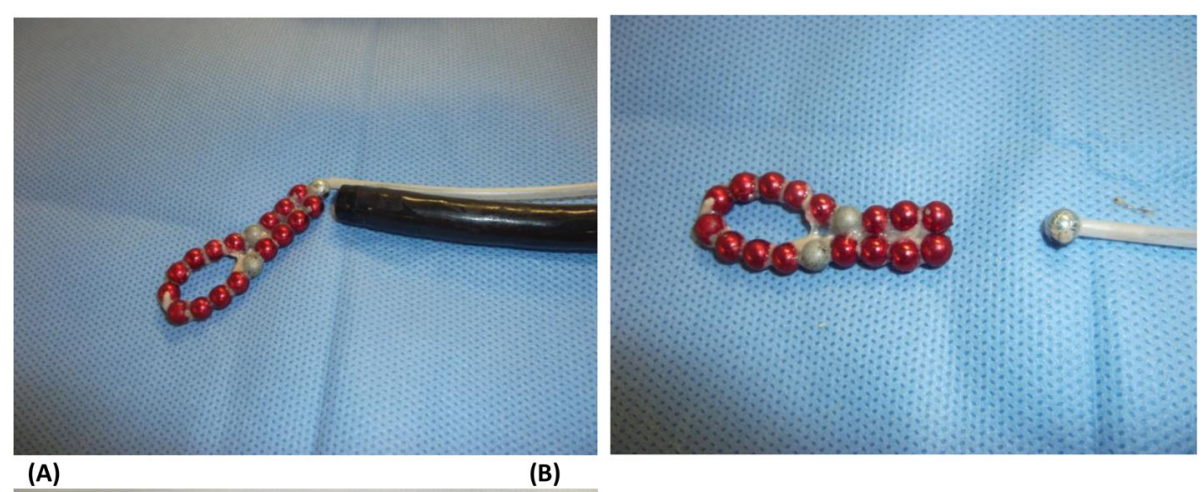

(B)

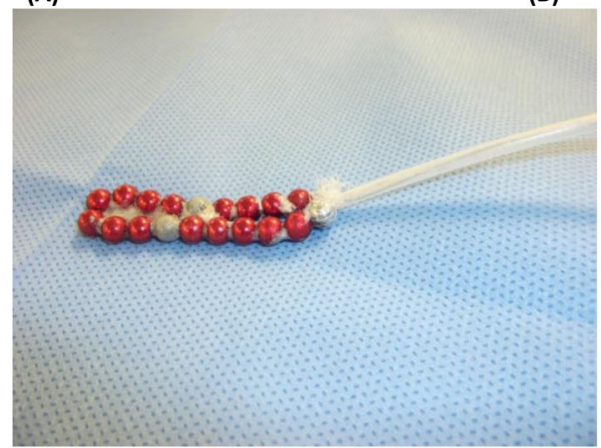

(C)

Fig. 5 A-C Simulation of the basic magnetic retriever attaching to the ingested Buckyball magnets alongside endoscopic vision

paediatric endoscope, into the stomach of the child where the ingested magnets were located (Fig. 4). Once the primitive magnetic retriever contacted the ingested magnets, they all united together to become one strong magnet and were brought out both quickly and safely under vision (Figs. 5 and 6). Of course, we could/should have passed the net through the endoscope before enclosing the magnet within its folds to create a single instrument.

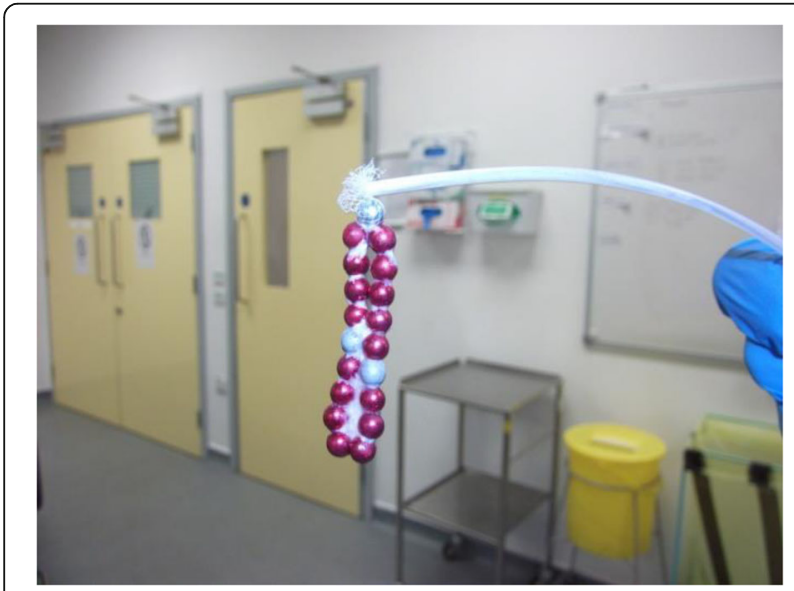

Fig. 6 Demonstration of the strong force upon which the basic magnetic retriever attaches to the ingested magnets
The images show a Buckyball magnet used within an endoscopic retrieval net to create the basic magnetic retriever. This was readily given to us by the parents. It is important to emphasise however that you can use any small magnet available as along as it could pass down easily through the oesophagus. The parents often do come however with a similar small magnet to the ones the child has swallowed and can easily be asked to help out by bringing one from home. It is our impression that both the magnet and the endoscopic retrieval net needed to replicate this basic magnetic retrieval are standard instruments that can be easily obtainable in any hospital. It is clearly important to test the magnet within the endoscopic retrieval net preoperatively to ensure that it stays secure within it and does not slip outside.

Simple magnetic retrievers are both easy to build and cost effective as they are built from basic equipment that, if not available, can be easily purchased in any children's theatre. It is also effective and time efficient in removing magnets from the stomach as it strongly and promptly attaches to the ingested magnets rendering it difficult to detach upon retrieval. Its safety also lies in the fact that the whole insertion and retrieval process with magnetic retriever here is done under endoscopic vision and hence can easily identify if uncoupling of the retrieved magnets occurred or if there is any gastric mucosal injury or bowel fistulation. Conventional endoscopic instruments are likely to be less effective than endoscopic-assisted 
magnetic retrievers in retrieving ingested metallic objects located in the stomach, due to weak bonds which form between the metal retrieval device and the magnet.

\section{Conclusion}

It is important to highlight the need for urgent removal of multiple ingested magnets or a single ingested magnet coupled with another ingested metallic object. Stricter implementation of a widespread ban over the use of loose magnets in toys is needed together with increasing public awareness of the hazards involved and the magnitude of this problem. Standardised protocols for the surgical management of ingested magnets should be available in every hospital.

\section{Acknowledgements}

Not applicable.

\section{Informed consent}

Retrospective informed consent was obtained before publication. IRB approval was not possible beforehand due to the emergency nature of the intervention required.

\section{Authors' contributions}

WE and EC contributed equally to collecting the data and researching and analysing the literature. WE wrote the initial manuscript and EC amended and approved the final manuscript. All authors have read and approved the final manuscript.

\section{Funding}

No funding to declare.

\section{Availability of data and materials}

All data generated or analysed during this study are included in this published article.

\section{Declarations}

Ethics approval and consent to participate

No ethical approval required.

\section{Consent for publication}

Patient's consent for publication was acquired.

\section{Competing interests}

The authors declare that they have no competing interests.

Received: 24 January 2021 Accepted: 10 September 2021

Published online: 01 December 2021

\section{References}

1. Mowry JB, Spyker DA, Cantilena LR, et al. 2012 Annual Report of the American Association of Poison Control Centers' National Poison Data System (NPDS): 30th Annual Report. Clin Toxicol (Phila). 2013;51(10):9491229. https://doi.org/10.3109/15563650.2013.863906.

2. Silverman JA, Brown JC, Willis MM, et al. Increase in pediatric magnetrelated foreign bodies requiring emergency care. Ann Emerg Med. 2013;62: 604-608.e1. https://doi.org/10.1016/j.annemergmed.2013.06.019.

3. Waters AM, Teitelbaum DH, Thorne V, Bousvaros A, Noel RA, Beierle EA. Surgical management and morbidity of pediatric magnet ingestions. I Surg Res. 2015;199(1):137-40. https://doi.org/10.1016/j.jss.2015.04.007.

4. BBC. Warning over fake piercing magnet craze. $h t t p s / / w w w . b b c . c o m / n e w s / u k-$ england-humber-43157923; Publ 22 Febr 2018, Last accessed 28 August 2018

5. Rosenfield D, Strickland M, Fecteau A. Magnet ingestion by a 3-year-old boy. Cmaj. 2013;185(11):972-4. https://doi.org/10.1503/cmaj.121847.

6. Consumers' Federation of Australia. ACT : Ban on small high powered magnets. http://consumersfederation.org.au/act-ban-on-small-highpowered-magnets/ 2012
7. Nui A, Hirama T, Katsuramaki T, Maeda T, Meguro M, Nagayama M, et al. An intestinal volvulus caused by multiple magnet ingestion: an unexpected risk in children. J Pediatr Surg. 2005;40(9):40-2. https://doi.org/10.1016/j. jpedsurg.2005.05.065.

8. Tavarez MM, Saladino RA, Gaines BA, Manole MD. Prevalence, clinical features and management of pediatric magnetic foreign body ingestions. J Emerg Med. 2013;44(1):261-8. https://doi.org/10.1016/j.jemermed.2012.03.025.

9. U.S. Consumer Product Safety Commission (2017, January). High-powered magnets and children make a deadly mix. Retrieved September 2021, from https://www.cpsc.gov: https://www.cpsc.gov/s3fs-public/High-Powered\%2 0Magnet\%20Safety\%20Alert.pdf.

10. United States Consumer Product Safety Comission. Buckyballs and Buckycubes high-powered magnet sets recalled due to ingestion hazard; Craig Zucker To Fund A Recall Trust, Settles With CPSC. https://www.cpsc.gov/content/buckyba Ils-and-buckycubes-high-powered-magnet-sets-recalled 2014.

11. Strickland $M$, Rosenfield $D$, Fecteau A. Magnetic foreign body injuries: a large pediatric hospital experience. J Pediatr. 2014;165(2):332-5. https://doi. org/10.1016/j.jpeds.2014.04.002.

12. Brown JC, Otjen JP, Drugas GT. Pediatric magnet ingestions: the dark side of the force. Am J Surg. 2014;207(5):754-9. https://doi.org/10.1016/j.amjsurg.2013.12.028.

13. North American Society for Pediatric Gastroenterology, Hepatology and Nutrition (2012, October 23). Warning Labels Ineffective at Preventing High-Powered Magnet Ingestions; NASPGHAN eleases New Survey Findings. Retrieved September 2021, from https://www.naspghan.org/files/documents/pdfs/advoca cy/2012/Final\%20NOLA\%20Press\%20Release.pdf.

14. Sola R, Rosenfeld EH, Yu YR, et al. Magnet foreign body ingestion: rare occurrence but big consequences. J Pediatr Surg. 2017. https://doi.org/10.1 016/j.jpedsurg.2017.08.013.

15. Dole M, Hiremath G. Successful endoscopic removal of 42 magnetic balls. J Dig Endosc. 2017;8:81-2. https://doi.org/10.4103/jde.JDE.

16. Blatt J, McLean TW, Castellino SM, et al. A review of contemporary options for medical management of hemangiomas, other vascular tumors, and vascular malformations. Pharmacol Ther. 2013;139(3):327-33. https://doi. org/10.1016/j.pharmthera.2013.05.001.

17. Equen M. A new magnet for foreign bodies in the food and air passages. J Am Med Assoc. 1945;127(2):87. https://doi.org/10.1001/jama.1945.92860020001009.

18. McDermott VGM, Taylor T, Wyatt JP, et al. Orogastric magnet removal of ingested disc batteries. J Pediatr Surg. 1995;30(1):29-32. https://doi.org/10.1 016/0022-3468(95)90602-9.

19. Yamauchi K, Kobayashi T, Shinomiya T, et al. Device for the removal of button batteries. Intern Med. 2001;40(1):9-13. https://doi.org/10.2169/internalmedicine.40.9.

20. Jaffe RB, Corneli HM. Fluoroscopic removal of ingested alkaline batteries. Radiology. 1984;150(2):585-6. https://doi.org/10.1148/radiology.150.2.6691 120.

21. Volle E, Beyer P, Kaufmann HJ, et al. Device for the removal of button batteries. J Dig Dis. 1945;13(1):87-32. https://doi.org/10.1016/0022-3468/9590602-9.

22. Diehl DL, Adler DG, Conway JD, et al. Endoscopic retrieval devices. Gastrointest Endosc. 2009;69(6):997-1003. https://doi.org/10.1016/j.gie.2009.01.005.

23. Kwong WT, Chang JT. Endoscopic retrieval of ingested nails using a refrigerator magnet. Clin Gastroenterol Hepatol. 2013;11(9):A24. https://doi. org/10.1016/j.cgh.2012.12.023.

24. Coash M, Wu GY. Endoscopic removal of a long sharp metallic foreign body by a snared magnet: an attractive solution. J Dig Dis. 2012;13(4):239-41. https://doi.org/10.1111/j.1751-2980.2012.00573.x.

25. Soong WJ, Yuh YS. Ingested button battery retrieved by a modified magnet endoscope. J Chinese Med Assoc. 2007;70(3):132-5. https://doi.org/10.1016/ S1726-4901(09)70344-2.

26. JK S. Endoscopic management of gastrointestinal foreign bodies in children. Indian J Pediatr 1999;66:S75-S80, 1 Suppl.

27. Nijhawan S, Joshi A, Shende A, Agarwal N, Kumar D, Mathur A, et al. Endoscopyassisted ferromagnetic foreign-body removal with a novel magnetic instrument. Endoscopy. 2004:36(12):1130. https:/doi.org/10.1055/s-2004-825980.

28. Nijhawan S, Rastogi M, Tandon M, Mallikarjun P, Singh V, Mathur A, et al. Magnetic loop basket: a 'two-in-one' instrument. Endoscopy. 2006;38(7): 723-5. https://doi.org/10.1055/s-2006-925454.

\section{Publisher's Note}

Springer Nature remains neutral with regard to jurisdictional claims in published maps and institutional affiliations. 